\title{
COLD FLOWS AND THE FIRST QUASARS
}

\author{
T. Di Matteo ${ }^{1}$, N. Khandai ${ }^{1}$, C. DeGraf ${ }^{1}$, Y. Feng $^{1}$, R. A. C. Croft ${ }^{1}$, J. Lopez ${ }^{2}$, And V. Springel ${ }^{3,4}$ \\ ${ }^{1}$ McWilliams Center for Cosmology, Carnegie Mellon University, 5000 Forbes Avenue, Pittsburgh, PA 15213, USA \\ ${ }^{2}$ Computer Science Department, Carnegie Mellon University, 5000 Forbes Avenue, Pittsburgh, PA 15213, USA \\ ${ }^{3}$ Heidelberg Institute for Theoretical Studies Schloss-Wolfsbrunnenweg 35, 68118 Heidelberg, Germany \\ ${ }^{4}$ Zentrum für Astronomie der Universität Heidelberg, Astronomisches Recheninstitut, Mönchhofstr. 12-14, 69120 Heidelberg, Germany \\ Received 2011 July 5; accepted 2011 December 5; published 2012 January 12
}

\begin{abstract}
Observations of the most distant bright quasars imply that billion solar mass supermassive black holes (SMBHs) have to be assembled within the first 800 million years. Under our standard galaxy formation scenario such fast growth implies large gas densities providing sustained accretion at critical or supercritical rates onto an initial black hole seed. It has been a long standing question whether and how such high black hole accretion rates can be achieved and sustained at the centers of early galaxies. Here we use our new MassiveBlack cosmological hydrodynamic simulation covering a volume $(0.75 \mathrm{Gpc})^{3}$ appropriate for studying the rare first quasars to show that steady high density cold gas flows responsible for assembling the first galaxies produce the high gas densities that lead to sustained critical accretion rates and hence rapid growth commensurate with the existence of $\sim 10^{9} M_{\odot}$ black holes as early as $z \sim 7$. We find that under these conditions quasar feedback is not effective at stopping the cold gas from penetrating the central regions and hence cannot quench the accretion until the host galaxy reaches $M_{\text {halo }} \gtrsim 10^{12} M_{\odot}$. This cold-flow-driven scenario for the formation of quasars implies that they should be ubiquitous in galaxies in the early universe and that major (proto)galaxy mergers are not a requirement for efficient fuel supply and growth, particularly for the earliest SMBHs.
\end{abstract}

Key words: cosmology: theory - galaxies: active - galaxies: evolution - galaxies: formation - hydrodynamics quasars: general

Online-only material: color figures

\section{INTRODUCTION}

It is now well established that the properties of supermassive black holes (SMBHs) found at the centers of galaxies today are tightly coupled to those of their hosts, implying a strong link between black hole and galaxy formation. The strongest constraint on the high-redshift evolution of SMBHs comes from the observations of the luminous quasars at $z \sim 6$ in the Sloan Digital Sky Survey (Fan et al. 2006; Jiang et al. 2009) and more recently at $z=7$ (Mortlock et al. 2011). Although rare (the comoving space density of $z \sim 6$ quasars is roughly $n \sim$ a few $\mathrm{Gpc}^{-3}$ ), the inferred hole masses of these quasars are in excess of $10^{9} M_{\odot}$ comparable to the masses of the most massive black holes in the universe today. The origin of these massive black hole seeds and the physical conditions that allow early growth to SMBHs remain a challenging problem.

In order to have sufficient time to buildup via gas accretion and mergers the first "seed" black holes must have appeared at an early epoch, $z>10$. The origin and nature of this seed population remain uncertain. Two distinct populations of $\mathrm{BH}$ seeds, in the range of $100-10^{5} M_{\odot}$, have been proposed: the small masses are usually thought to be the remnants of the first generation of PopIII stars formed of metal-free gas at $z \sim 20$-30 (e.g., Bromm et al. 1999; Abel et al. 2000; Nakamura \& Umemura 2001; Yoshida et al. 2003; Gao et al. 2005), while the large seeds form in direct dynamical collapse in metalfree galaxies (Koushiappas et al. 2004; Begelman et al. 2006, although see also Mayer et al. 2010 for direct collapse into a massive black hole in the metal-enriched regime).

Growing seeds to $10^{9} M_{\odot}$ in less than a billion years requires extremely large accretion rates. For a black hole accreting at the critical Eddington accretion rate its luminosity $L_{\mathrm{Edd}}=$ $\left(4 \pi G c m_{p}\right) / \sigma_{T} M_{\mathrm{BH}}=\eta \dot{M}_{\mathrm{Edd}} c^{2}$ (where $G, c, m_{p}$, and $\sigma_{T}$ are the gravitational constant, speed of light, proton mass, and Thomson cross section, and $\eta$ is the standard accretion efficiency) implies an exponential growth at the characteristic Eddington timescales, $t_{\text {Edd }}=450 \eta /(1-\eta)$ Myr, such that $M_{\mathrm{BH}}=M_{\text {seed }} e^{t / t_{\mathrm{Edd}}}$. For a seed mass ranging from $M_{\text {seed }} \sim$ 100 to $10^{5} M_{\odot}$ this requires $10-17 e$-foldings to reach $M_{\mathrm{BH}} \sim$ $10^{9} M_{\odot}$. The crucial question (for any given seed model) is then where (if at all, in which kind of halos) and how (at what gas inflow rates) such vigorous accretion can be sustained at these early times.

As bright quasars likely occur in rare high-density peaks in the early universe, large computational volumes are needed to study them. Here we use a new large cosmological smooth particle hydrodynamics ( $\mathrm{SPH}$ ) simulation, MassiveBlack (covering a volume of $[0.75 \mathrm{Gpc}]^{3}$ ), with sufficiently high resolution (over 65 billion particles) to be able to include tested prescriptions for star formation, black hole accretion, and associated feedback processes to investigate whether and if such objects may be formed within our standard structure formation models. Crucially, our MassiveBlack simulation is of sufficiently high resolution to allow us to follow the mass distribution in the inner regions of galaxies while still evolving a close to Gigaparsec scale region. It therefore provides a unique framework to study the formation of the first quasars.

\section{METHODOLOGY}

\subsection{Simulation Run: "MassiveBlack"}

Our new simulation has been performed with the cosmological TreePM-SPH code P-GADGET, a hybrid version of GADGET2 (Springel 2005) which has been extensively modified and upgraded to run on the new generation of Petaflop supercomputers. The major improvement over previous 
versions of GADGET is in the use of threads in both the gravity and SPH part of the code which allows the effective use of multi-core processors combined with an optimum number of MPI tasks. Here, we present results from the largest simulation to date which was run on $10^{5}$ cores corresponding to the entire Cray-XT5 "Kraken" at NICS. The MassiveBlack simulation contains $N_{\text {part }}=2 \times 3200^{3}=65.5$ billion particles in a volume of $533 \mathrm{Mpc}^{-1}$ on a side with a gravitational smoothing length $\epsilon=5 \mathrm{kpc} h^{-1}$ in comoving units). The gas and dark matter particle masses are $m_{\mathrm{g}}=5.7 \times 10^{7} M_{\odot} h^{-1}$ and $m_{\mathrm{DM}}=2.8 \times 10^{8} M_{\odot} h^{-1}$, respectively. This run contains gravity and hydrodynamics but also extra physics (subgrid modeling) for star formation (Springel \& Hernquist 2003), black holes, and associated feedback processes. The simulation has currently been run from $z=159$ to $z=4.75$ (beyond our original target redshift of $z=6$ ). For this massive calculation it is currently prohibitive to push it to $z=0$ as this would require an unreasonable amount of computational time. The redshift range probes early structure formation and the emergence of the first galaxies and quasars. We use WMAP5 (Dunkley et al. 2009) cosmological parameters (flat, $\Omega_{m}=0.24, \sigma_{8}=0.8$, and $h_{0}=0.72$ ).

\subsection{Black Hole Accretion and Feedback Model}

The prescription for accretion and associated feedback from massive black holes has been developed by Di Matteo et al. (2005) and Springel et al. (2005). Detailed studies of this implementation in cosmological simulations and associated predictions (Sijacki et al. 2007; Li et al. 2007; Di Matteo et al. 2008; Croft et al. 2009; Sijacki et al. 2009; Colberg \& di Matteo 2008; DeGraf et al. 2010, 2011c; Booth \& Schaye 2011) have shown that it can reproduce all the basic properties of black hole growth, the observed $M_{\mathrm{BH}}-\sigma$, relation (Di Matteo et al. 2008), the quasar luminosity function (DeGraf et al. 2010), and its evolution as well as the spatial clustering of quasars (DeGraf et al. 2011a, 2011c). Because of the extensive literature on this model we here only give the major features and refer the reader to the above for more details and resolution tests. There is absolutely no variation in parameters or any feature in the model used here.

In a nutshell our black hole accretion and feedback model (Di Matteo et al. 2008) consists of representing black holes by collisionless particles that grow in mass (from an initial seed black hole) by accreting gas in their environments. A fraction of the radiative energy released by the accreted material is assumed to couple thermally to nearby gas and influence its motion and thermodynamic state (BH feedback). The model should be viewed in the context of cosmological growth of black holes and not detailed $\mathrm{BH}$ accretion physics. Resolution tests have been performed extensively in the literature and here we are within our converged regime (Di Matteo et al. 2008).

We introduce collisionless "sink" particles in the simulations to model black holes at the centers of forming minihalos that do not have one already (we use Friends-of-Friends on the fly). We place a black hole seed of fixed mass, $M=10^{5} h^{-1} M_{\odot}$. The black hole particle then grows in mass via accretion of surrounding gas according to $\dot{M}_{\mathrm{BH}}=\left(4 \pi G^{2} M_{\mathrm{BH}}^{2} \rho\right) /\left(\left(c_{s}^{2}+\right.\right.$ $\left.v^{2}\right)^{3 / 2}$ ) (where $\rho$ and $c_{s}$ are the density and sound speed of the hot and cold phase of the Interstellar Medium (ISM) gas which were taken into account appropriately as in Pelupessy et al. (2007) and $v_{\mathrm{BH}}$ is the velocity of the black hole relative to the gas) and by merging with other black holes. We limit the accretion rate to Eddington.
The radiated luminosity, $L_{\mathrm{r}}$, from the black hole is related to the accretion rate, $\dot{M}_{\mathrm{BH}}$ as $L_{\mathrm{r}}=\epsilon_{\mathrm{r}}\left(\dot{M}_{\mathrm{BH}} \times c^{2}\right)$, where we take the standard mean value $\epsilon_{\mathrm{r}}=0.1$. Some coupling between the liberated luminosity and the surrounding gas is expected: in the simulation $5 \%$ of the luminosity is (isotropically) deposited as thermal energy within its softening length, providing some form of feedback (Di Matteo et al. 2005). Two black hole particles merge if they come within the spatial resolution (i.e., within the local SPH smoothing length) with relative speed below the local sound speed.

Independent works, and in particular, the cosmological simulations by Booth \& Schaye (2009) and Johansson et al. (2008) have explored the parameter space of the reference model of Di Matteo et al. (2008), as well as variations of our model prescriptions. This large body of existing work and investigations make this particular model a good choice for more detailed studies of the growth of the first quasars which is the subject we focus on here. In associated publications we show that the MassiveBlack quasars are fully consistent with all the statistical constraints for the observed populations of high redshift quasars, such as the observed luminosity functions of and clustering of quasars (DeGraf et al. 2011b) as well as properties of quasar hosts (Khandai et al. 2011). To produce the black hole history from the simulation data we rely on SQL databases developed by Lopez et al. (2011).

\section{RESULTS}

The cosmological gas density distribution in the full volume of the MassiveBlack is shown in the large scale image of Figure 1. The panel shows the whole of the three-dimensional simulation volume "unwrapped" into a two-dimensional image slice (Feng et al. 2011) at $z=5$. At these large scales the density distribution of the universe appears fairly uniform. The resolution however is sufficiently fine to make it possible to zoom into increasingly smaller regions and search for massive black holes that have experienced significant growth. Superimposed onto the full scale image we show a zoomed region (scale of $100 \mathrm{Mpc} h^{-1}$ and $10 \mathrm{Mpc} h^{-1}$ on a side from left to right, respectively) around one of the largest black holes/quasars at this time. On these scales the images show the typical filaments that compose the cosmic web and in particular how the first massive quasars (the most massive black hole, at the center, has a mass of $M_{\mathrm{BH}} \sim 3 \times 10^{9} M_{\odot}$ ) form at the same type of intersection/nodes of filaments that are the expected locations of rare (massive) dark matter halos. Remarkably, we do find 10 black holes in the volume that have grown to about a billion solar masses by $z \sim 6$ or earlier (and many other of smaller masses).

Figure 2 shows the environment and its evolution (five time lapses from about $z \sim 8$ to $z \sim 5$ ) of three examples from within this sample. Their detailed mass assembly history is shown in Figure 3. The panels in Figure 2 show the evolution of the gas density color coded by temperature. These objects are found to be continuously fed by intense streams of high density gas (this consistent with the cold-accretion picture for the growth of galaxies at intermediate/high redshift by, e.g., Dekel \& Birnboim (2006). To illustrate the origin of the gas that eventually ends up in the $\mathrm{BH}$ accretion region we track the position of particles that are eventually accreted by the $\mathrm{BH}$ at $z=5,6$ (white points in Figure 2). During these times Eddington accretion is attained and sustained (Figure 3). By showing the temperature of the gas, the images clearly make 


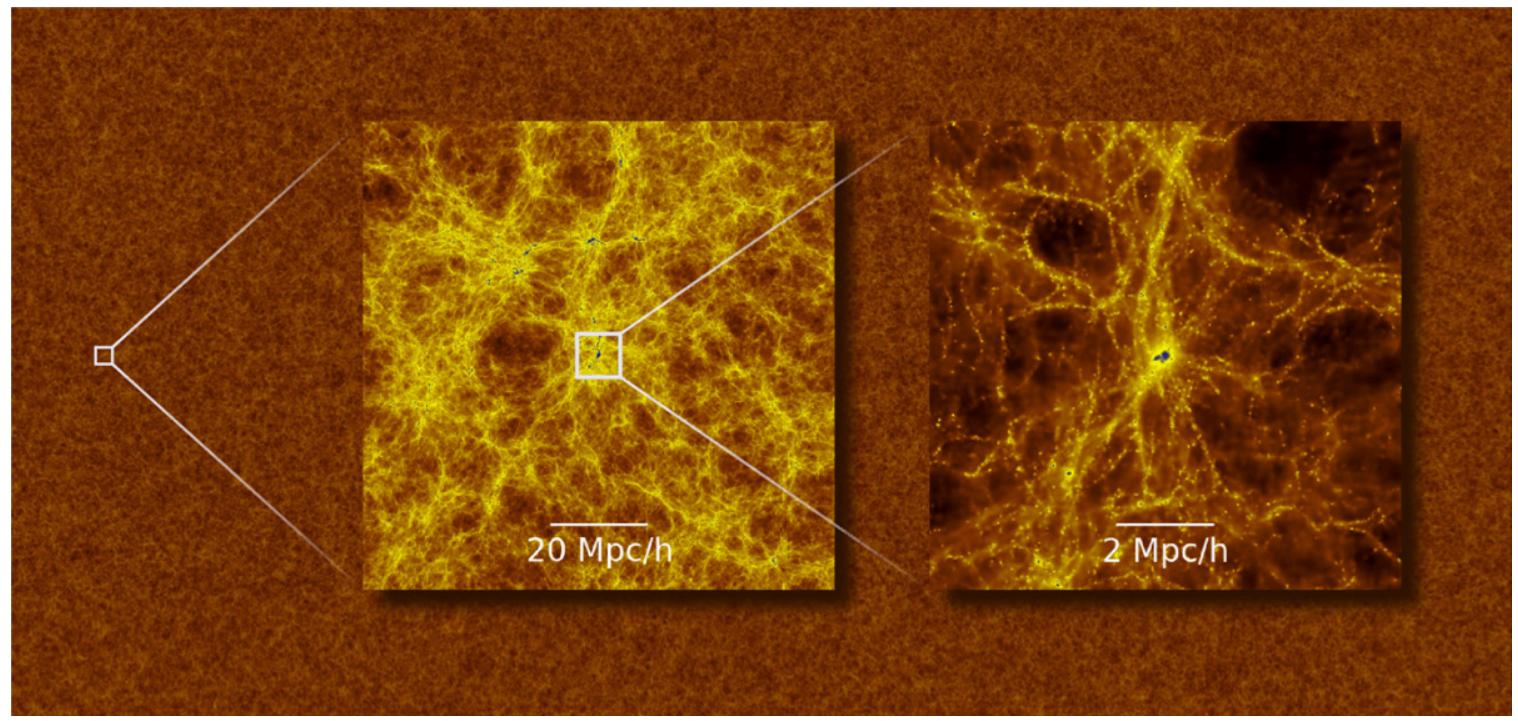

Figure 1. Projected gas density over the whole volume ("unwrapped" into two-dimensional) is shown in the large scale image at $z=5$. The two overlaid panels show successive zoom-ins by a factor of 10, centered on the region of the most massive BH. The dark color at high densities represents star-forming gas (Feng et al. 2011). (A color version of this figure is available in the online journal.)
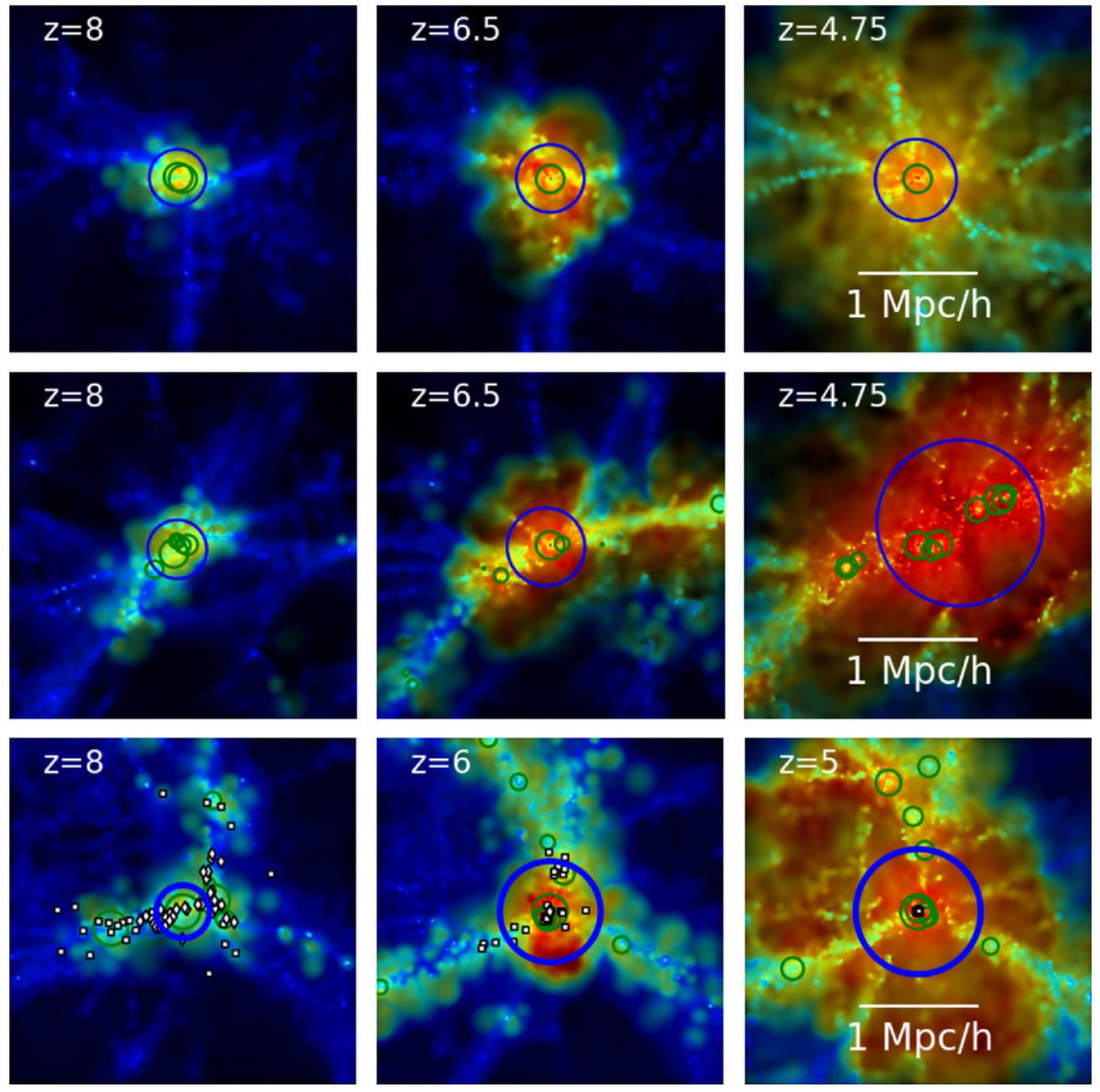

Figure 2. Projected gas distribution color coded by temperature around three example quasars (one in each row) across five different redshifts (labeled on each of the five panels; left to right). The projected density ranges from $\sim 10^{-2}$ to $\sim 10^{2} h M_{\odot} \mathrm{pc}^{-2}$ and the temperature from $\sim 10^{4}$ (blue colors) to $10^{8} \mathrm{~K}$ (red colors). The quasar positions are indicated by the green circles and the virial radius of the halo by the blue circles. The white points in the bottom panel show particles that are eventually accreted onto the $\mathrm{BH}$ at $z=5,6$.

(A color version of this figure is available in the online journal.) 

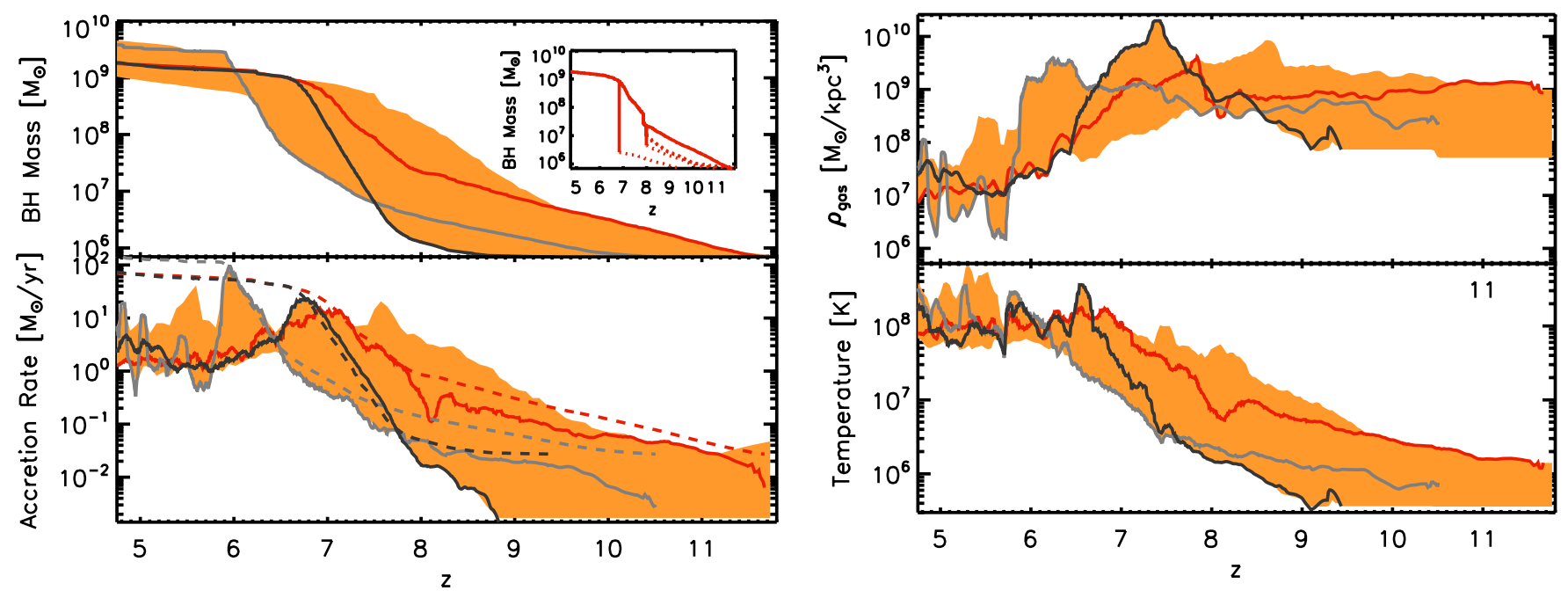

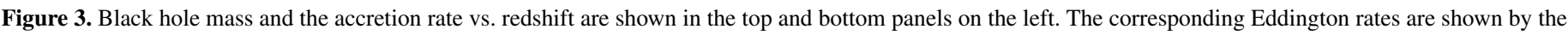

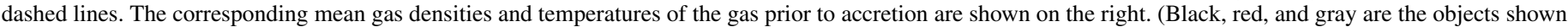

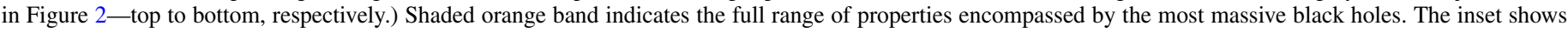
an example of $\mathrm{BH}$ mergers onto the main (red) progenitor.

(A color version of this figure is available in the online journal.)

visible an expanding "bubble" (emerging from about $z \sim 6.5-7$ ) of hot gas (red colors) around the central quasars (whose positions are indicated by green circles). This bubble created by the $\mathrm{BH}$ feedback is more or less confined within the halo (the virial radius of the halo is shown by the blue circles in Figure 2) for $z \lesssim 6$. Below this redshift, and rather abruptly, the energy released by the quasars heats and expels the gas as a wind well beyond the halo. Black hole growth has now become self-regulated (see also Figure 3, accretion levels off below Eddington). Although the effects of quasar feedback in our model have been studied in detail previously (Di Matteo et al. 2005, 2008) what is remarkable here is that even though feedback energy consistently heats the gas within and eventually beyond the scale of halos, it does not do much to the streams of inflowing cold gas. The streams get somewhat (albeit not completely) disrupted only at $z \lesssim 6$. Before this happens billion solar mass black holes are already assembled (see Figure 3), a process that takes a few hundred million years.

Sustained phases of Eddington accretion onto these black holes start as early as $z \sim 9-10$ and go on uninterrupted until $z \sim 6-7$, leading to BH masses of the order of $10^{9} M_{\odot}$ in the first massive halos of roughly $M_{\text {halo }} \sim 10^{12} M_{\odot}$ at $z \sim 6-7$ (rare $3 \sigma-4 \sigma$ peaks of the density distribution). We have indeed a few objects (the range of the 10 most massive is shown by the orange areas in Figure 3) that reach these high masses already at $z \sim 7$ consistent with Mortlock et al. (2011). Interestingly, this process of BH assembly is apparently facilitated by the "cold flows" picture of galaxy formation below and at the threshold dark matter mass of $M_{\text {halo }} \sim 10^{12} M_{\odot}$ (Dekel \& Birnboim 2006; Dekel et al. 2009; Kereš et al. 2005, 2009). With MassiveBlack we are able to trace the formation of the first, rare massive halos, those which are mostly assembled by high density (high redshift) cold streams (Dekel et al. 2009). We find that these same streams easily penetrate all the way into central regions of galaxies even in the presence of strong feedback. We find however that once $M_{\text {halo }} \sim 10^{12} M_{\odot}$ and halos enter the so-called shock-heated regime (Dekel et al. 2009) the BH growth levels off as it becomes finally self-regulated (Figure 3). The temperature (average over the two-phase ISM) of the accreting gas also is raised well above the virial temperature, $T_{\mathrm{vir}} \sim 10^{7} \mathrm{~K}$, rendering some of the gas unbound (Figure 3). Black hole masses level off at a few $10^{9} M_{\odot}$ (Figure 3). Even though at $z<6$ in massive halos the outflows clearly affect the incoming gas if they do not fully disrupt the cold flows.

We note that the black holes occupying these rare massive halos hardly undergo any major mergers, indicating that their hosts are not undergoing major galaxy mergers (even though minor mergers of subhalos/clumps are however common). In the inset in Figure 3 we show the histories of all black holes that merge into the main progenitor BH shown in red, which is typical of what we see: a few minor mergers occur. At the redshifts relevant for the first quasars, major mergers are still extremely rare events and halos have not yet assembled above their shock-heating scale (Dekel et al. 2009), so that quasars are fueled directly by cold streams/flows.

To illustrate the origin of the gas growing the first quasars we track in the simulation the temperature history of particles that end up being accreted onto the BH. Figure 4 shows one example of the temperature as a function of proper radius of particles that at $z=6,7$ (circles and squares, respectively) have participated in $\mathrm{BH}$ accretion. We choose these two redshifts to track particles from because they are at the peak of the accretion rate $(z=7)$ and below it $(z=6$; for the object shown in the middle panel of Figure 2 and shown as in red in Figure 3). In this plot, all the particles' positions are tracked back all the way to $z=8.5$ so to show their temperature evolution as they enter the halo and travel within the BH smoothing length.

The plot shows that the great majority of particles that participate in the black hole growth enter cold $\left(T_{\text {gas }} \sim 10^{4} \mathrm{~K}\right)$ and remain below $T_{\text {gas }} \sim 10^{5.5-6} \mathrm{~K}$ well into the tens of kpc region just as predicted for gas participating in cold flows by Dekel \& Birnboim (2006) and Kereš et al. (2005). In the shockheated regime the temperature of the gas would rise as it enters the halo. At the smallest scales the gas (particles in the two-phase medium are included) is significantly heated by the black hole feedback. This is consistent with what shown in the mean (over the two-phase ISM) temperature of the gas prior to accreting onto the BH in Figure 3. 


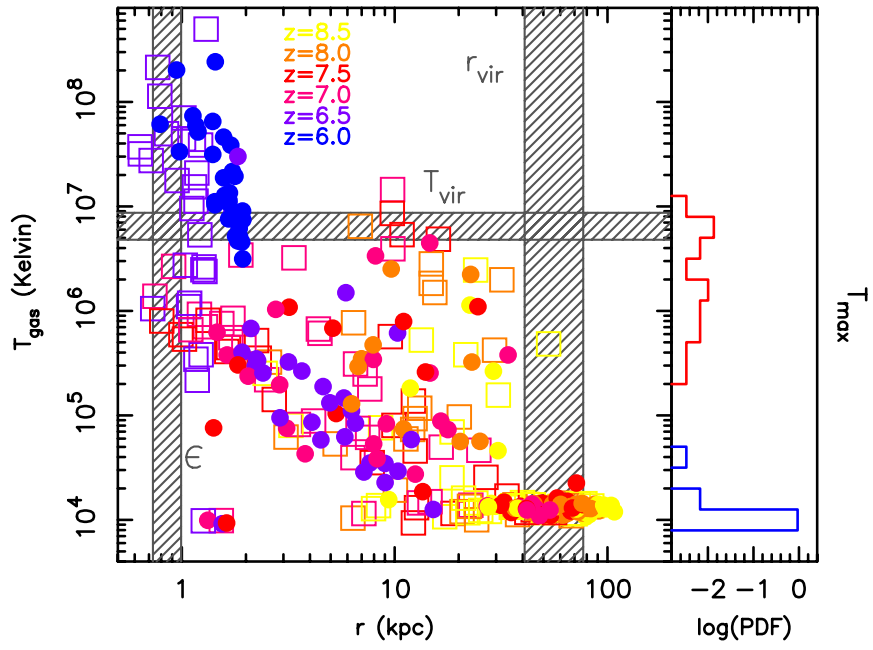

Figure 4. Temperature of the 32 nearest $\mathrm{BH}$ neighbors (gas particles actively participating in $\mathrm{BH}$ accretion) at $z=6$ (solid points) and $z=7$ (squares) vs. position tracked back to $z=8.5$ ( $r$ is in physical units). This example is for second halo in Figure 2. The rightmost vertical shaded area shows the evolution of $r_{\text {vir }}$ with the horizontal one the corresponding temperature $T_{\text {vir }}$ and $\epsilon$ is the gravitational smoothing length from $z=6$ to 8.5. On the side, the histogram shows the maximum temperature of all gas particles that enter the $\mathrm{BH}$ smoothing length from $z=12$ to $z=5$ (prior to participating in the two-phase medium).

(A color version of this figure is available in the online journal.)

To quantify more directly the nature of the gas that grows the black holes we plot a histogram of its maximum temperature (in this case prior to participating in the two-phase medium and hence being affected by various forms of feedback) of the gas particles at any time between $z=12$ and $z=5$ (right side of Figure 4) that end up in the $\mathrm{BH}$ accretion region. Over $95 \%$ of the gas responsible for growing the $\mathrm{BH}$ is always at $T_{\text {gas }} \sim 10^{4} \mathrm{~K}$ with the remaining fraction possibly shock-heated $\left(10^{5} \mathrm{~K}<T<10^{7} \mathrm{~K}\right)$.

Indeed, when we trace the positions of the particles that are in the $\mathrm{BH}$ accretion region at $z=5$ and $z=6$ for the same black hole (white points in bottom panel of Figure 2), even past the time of major phase of $\mathrm{BH}$ growth all these particles originate straight from the cold streams (and subhalos) of gas within the large scale filaments rather than a spherical hot medium from which they cool or a merger with a similar size halo.

\section{CONCLUSIONS}

With our new large cosmological simulation MassiveBlack we show that the short timescale associated with infall via cold flows and the short cooling timescales in cold radial streams that penetrate the halo render the flow into the central regions unstoppable by feedback, allowing it to easily sustain $\mathrm{BH}$ growth at the Eddington rates to build up the required $\mathrm{BH}$ masses by $z=6-7$. One consequence of this scenario is that $\mathrm{BH}$ masses at these redshifts are expected to show deviations from the local $M_{\mathrm{BH}}-\sigma$ relation. $\mathrm{BH}$ masses assembled faster (most growth occurs over few hundred million years) than the stellar spheroid (assembled over Gyr timescales) as suggested by some observations. Stream fed accretion will still be relevant over the peak of the quasar phase but mergers (whose rates peak closer to those redshifts) will become an increasingly major player in their growth. We speculate however that most of the growth of a quasar's mass will always occur before the shock-heating scale of an halo is reached much like most of its star formation rate (Dekel et al. 2009). We will investigate this further in our large volume in future work. Our scenario is somewhat similar to that proposed by Mayer et al. (2010) or Li et al. (2007), however, crucially, it does not rely on a major merger to induce the strong gas inflows but points to a more common origin for them particularly at these redshifts (which we could find by having a large volume simulation). As we have shown, relaxing the constraint for massive mergers makes it plausible to attain quite commonly large black hole masses as high as $z=7$ commensurate with Mortlock et al. (2011).

This research has been supported by NSF AST 1009781, NSF OCI 0749212, and Urania-E. Stott. Computations were performed at NICS. We acknowledge the Aspen Center for Physics. The simulations are uploaded on GIGAPAN and be viewed on http://www.gigapan.org/gigapans/76215/.

\section{REFERENCES}

Abel, T., Bryan, G. L., \& Norman, M. L. 2000, ApJ, 540, 3 i9

Begelman, M. C., Volonteri, M., \& Rees, M. J. 2006, MNRAS, 370, 289

Booth, C. M., \& Schaye, J. 2009, MNRAS, 398, 53

Booth, C. M., \& Schaye, J. 2011, MNRAS, 413, 1158

Bromm, V., Coppi, P. S., \& Larson, R. B. 1999, ApJ, 527, L5

Colberg, J. M., \& di Matteo, T. 2008, MNRAS, 387, 1163

Croft, R. A. C., Di Matteo, T., Springel, V., \& Hernquist, L. 2009, MNRAS, 400, 43

DeGraf, C., Di Matteo, T., \& Springel, V. 2010, MNRAS, 402, 1927

DeGraf, C., Di Matteo, T., \& Springel, V. 2011a, MNRAS, 413, 1383

DeGraf, C., Di Matteo, T., Khandai, N., et al. 2011b, MNRAS, submitted (arXiv:1107.1254)

DeGraf, C., Oborski, M., Di Matteo, T., et al. 2011c, MNRAS, 416, 1591

Dekel, A., \& Birnboim, Y. 2006, MNRAS, 368, 2

Dekel, A., Birnboim, Y., Engel, G., et al. 2009, Nature, 457, 451

Di Matteo, T., Colberg, J., Springel, V., Hernquist, L., \& Sijacki, D. 2008, ApJ, 676,33

Di Matteo, T., Springel, V., \& Hernquist, L. 2005, Nature, 433, 604

Dunkley, J., Komatsu, E., Nolta, M. R., et al. 2009, ApJS, 180, 306

Fan, X., Strauss, M. A., Becker, R. H., et al. 2006, AJ, 132, 117

Feng, Y., Croft, R., Di Matteo, T., et al. 2011, ApJS, 197, 18

Gao, L., Able, T., Frenk, C. S., et al. 2005, MNRAS, 363, 379

Jiang, L., Fan, X., Bian, F., et al. 2009, AJ, 138, 305

Johansson, P. H., Naab, T., \& Burkert, A. 2008, Astron. Nachr., 329, 956

Kereš, D., Katz, N., Fardal, M., Davé, R., \& Weinberg, D. H. 2009, MNRAS, 395,160

Kereš, D., Katz, N., Weinberg, D. H., \& Davé, R. 2005, MNRAS, 363, 2

Khandai, N., Di Matteo, T., Croft, R. A. C., \& Springel, V. 2011, MNRAS, in press (arXiv:1111.0692)

Koushiappas, S. M., Bullock, J. S., \& Dekel, A. 2004, MNRAS, 354, 292

Li, Y., Hernquist, L., Robertson, B., et al. 2007, ApJ, 665, 187

Lopez, J., DeGraf, C., DiMatteo, T., et al. 2011, in Proc. Conf. Twenty-third Scientific and Statistical Database Management, http://www.pdl.cmu.edu/ PDL-FTP/AstroDISC/disc-holes_abs.shtml

Mayer, L., Kazantzidis, S., Escala, A., \& Callegari, S. 2010, Nature, 466, 1082 Mortlock, D. J., Warren, S. J., Venemans, B. P., et al. 2011, Nature, 474, 616

Nakamura, F., \& Umemura, M. 2001, ApJ, 548, 19

Pelupessy, F. I., Di Matteo, T., \& Ciardi, B. 2007, ApJ, 665, 107

Sijacki, D., Springel, V., di Matteo, T., \& Hernquist, L. 2007, MNRAS, 380, 877

Sijacki, D., Springel, V., \& Haehnelt, M. G. 2009, MNRAS, 400, 100

Springel, V. 2005, MNRAS, 364, 1105

Springel, V., Di Matteo, T., \& Hernquist, L. 2005, MNRAS, 361, 776

Springel, V., \& Hernquist, L. 2003, MNRAS, 339, 289

Yoshida, N., Sokasian, A., Hernquist, L., \& Springel, V. 2003, ApJ, 598, 73 